\title{
Assessment of psychiatrists' approaches regarding disclosure of psychiatric disorders to their patients: a qualitative study
}

\author{
Anahita Amidi Naeini ${ }^{1}$, Hadi Ranjbar ${ }^{2}$, Homa Mohammadsadeghi ${ }^{2}$, Kaveh Alavi ${ }^{2}$, Hamidreza Ahmadkhaniha², \\ Maryam Rasoulian*2 (D)
}

Received: 19 Jan 2019

Published: 20 Jul 2020

\section{Abstract}

Background: Diagnosis disclosure is the result of a shift in medical approaches from traditional paternalism to participatory and patient-centered decision making. Disclosure of psychiatric diagnosis remained uncommon and controversial. Giving information about psychiatric illnesses is very complicated, and it is affected by several factors. While clinical guidelines provide a clear pathway for treating patients, in practice, the treatment of patients is influenced by cultural and social factors. The aim of the current study was a qualitative assessment of psychiatrists' approaches regarding the disclosure of psychiatric disorders to their patients.

Methods: The current study was conducted with a qualitative approach. The participants were purposefully selected psychiatrists from three medical universities in Tehran, Iran. The data gathered using the semi-structured interview method. Sixteen interviews with 14 psychiatrists were conducted. Data were analyzed using thematic analysis.

Results: Psychiatrists decide to disclose the diagnosis based on several factors. We summarized these factors in a central theme, passive situational decision making based on paternalism and displacement of responsibility. It has two subthemes, including "passive and situational decision making" and "paternalism and displacement of responsibility." Each theme presented by detailed quotations.

Conclusion: The results of this study showed that psychiatrists did not actively disclose the diagnosis name to patients. Diagnosis disclosure was influenced by several factors, such as the certainty about the diagnosis and the severity of the disease. This passive approach does not respect the patient's rights. The paternalistic nature of this approach mandates psychiatrists to consider themselves as the responsible perosn for their patients' welfare.

Keywords: Disclosure, Diagnosis, Psychiatrists, Mental health, Medical ethics

Conflicts of Interest: None declared
Funding: None

*This work has been published under CC BY-NC-SA 1.0 license.

Copyright $\odot$ Iran University of Medical Sciences

Cite this article as: Amidi Naeini A, Ranjbar H, Mohammadsadeghi H, Alavi K, Ahmadkhaniha H, Rasoulian M. Assessment of psychiatrists' approaches regarding disclosure of psychiatric disorders to their patients: a qualitative study. Med J Islam Repub Iran. 2020 (20 Jul);34:82. https://doi.org/10.47176/mjiri.34.82

\section{Introduction}

Diagnosis disclosure is the result of a shift in medical approaches from traditional paternalism to participatory and patient-centered decision making. The protection of

Corresponding author: Dr Maryam Rasoulian, rasoulian.m@iums.ac.ir

1. Medical School, Iran University of Medical Sciences, Tehran, Iran

2. Mental Health Research Center, Psychosocial Health Research Institute, Iran University of Medical Science, Tehran, Iran the patient's rights is the foundation of this shift. In the participatory and patient-centered approaches, the patient is an active member in the process of treatment (1-3). As a

$\uparrow$ What is "already known" in this topic:

The disclosure of diagnosis to patients is one of the most important principles in respecting their rights. Psychiatrists face many challenges in disclosing psychiatric diagnoses to their patients.

$\rightarrow$ What this article adds:

Psychiatrists followed a medical model of diagnosis disclosure which emphasizes the reduction of symptoms. They did not disclose the diagnosis to their patients as a part of the treatment process. Diagnosis disclosure was influenced by several factors such as the certainty about the diagnosis and the severity of the disease. 
result, patients receive all needed information, and they make decisions related to their treatment. There is a presumption in health service delivery systems that physicians are required to give patients complete information about their condition (4). While some freedom is given to doctors about the time and amount of data that they give patients; it is no longer assumed that they can provide the information only with the patient's request (5). The participatory approach takes more into account the benefits of the patient as they gain more access to knowledge, resources, and treatment options (2).

Despite the above mentioned, disclosure of psychiatric diagnosis remained uncommon and controversial. It has also been the subject of studies for a long time. The results of the researches showed that psychiatrists do not tend to disclose the diagnosis or they delay it. For example, in a study by Shergill, Barker (6), more than half of the 126 psychiatric patients were unaware of their diagnosis. Even among different illnesses, the level of diagnosis disclosure is different. In the study of McDonald-Scott, Machizawa (7), the results showed that the disclosure of borderline diagnosis was significantly more than schizophrenia. Also, the results of researches in different countries showed that disclosure rates were different in different countries. The percentage of disclosure of schizophrenia was different in Japan and the United States. More recent studies have shown a higher degree of disclosure of schizophrenia. In a study in Scotland, 59 percent of schizophrenic patients knew their diagnosis (8-10).

Giving information about psychiatric illnesses is very complicated, and it is affected by several factors. The name of the diagnosis, the possibility of making distress for the patient, patient insight, understanding the outcome of the disease, agreement over treatments, and prevention of stigma for the patient are effective factors in diagnosis disclosure $(11,12)$. While poor insight which can be the result of nondisclosure of diagnosis is one of the predictors of non-adherence to treatment, diagnosis disclosure remained very uncommon in psychiatric settings $(13,14)$. The patient can not accept treatment until he/she believes in his/her disease. Poor insight is also related to a worse course of illness, such as increasing the number of attacks and hospitalization. It also results in functional failure, poor social skills, and lower social relationships. If the patient does not know the diagnosis, many problems arise; one of these problems is the lack of adherence to the treatment (15-17).

When a patient does not know the diagnosis places $\mathrm{him} / \mathrm{her}$ in a difficult situation that can not explain his or her own circumstances or find the right explanation for what is happening. In a study done by Marzanski (18), dementia patients were asked if they knew the name of their disease, who told them the name of the disease and what they need to know. Their results showed that patient information was deficient and most of them tended to have more information. However, the results showed that a number of patients did not want to know their diagnosis, and the rights of these patients should also be preserved.

From the perspective of medical ethics, full disclosure should be made to respect patients' rights. However, the disclosure of psychiatric diagnoses is controversial. The experience of the research team shows that many psychiatric patients do not know their diagnosis. While clinical guidelines provide a clear pathway for treating patients, in practice, the treatment of patients is influenced by cultural and social factors. The aim of the current study was to assess psychiatrists' approaches on disclosure of psychiatric disorders to their patients.

\section{Methods}

The current study was conducted with a qualitative approach. The participants were purposefully selected psychiatrists who had work experience for more than five years in the field of psychiatry. The list of psychiatrists with experience of more than five years was received from the Iranian Psychiatrists' Association. The initial participants were from the experienced psychiatric professors working in university-affiliated hospitals of Tehran. The sampling continued with psychiatrists within different sexes. Then, to reach the maximum variation of the study sample, the sampling continued by choosing psychiatrists from both sexes, with different age and work experiences. Participants from the private sector and psychiatrists with various treatment approaches (psychanalytic and biopsychosocial) also were selected in the process of data gathering to answer the questions that rise through data analysis.

The data gathered using semi-structured interview method. The corresponding author who is a psychiatrist and a university professor, did the interviews. The first author (psychiatrist) and the second author (Ph.D. in Nursing) were present in some interviews. The research team developed an interview guide for first interviews. The interview guide included three sections including initial questions such as "can you explain your method of diagnosis disclosure?". The central part of the interview consists of intermediate questions such as "what was your patient reaction?" or "how do you manage your patient reactions?". In the end, the interview was closed with ending questions like "do you have anything to add?". In the process of data gathering, new questions were raised from data analysis and were added to the guideline. For example, when the analysis showed that the experience of psychiatrists in the private sector might be different from ones in the public sector, some interviews were conducted with that group. Interviews were conducted where the interviewees were comfortable, mostly in their office.

Data were analyzed using thematic analysis as it demonstrated by Braun and Clarke (19). The first step was familiarizing with data. The first author transcribed all interviews and read them several times. The second step was generating initial codes. In this step, all authors participated in the coding process. The first author coded interviews in a file with Microsoft word format and emailed them to all members of the team. All members of the research team reviewed the coded segments. Each interview was sent to the related participant to comment on the codes. The third step was searching for themes. From the first interview, similar codes were gathered to form primary categories. Categories and sub-categories became more specific and more advanced as interviews 
progressed. In this step, we searched for patterns in data to form our themes by connecting categories to each other. The patterns that identified in the data formed the primary themes. In the fifth step primary themes were reviewed and two main themes were chosen. In the sixth step, the selected themes were named. After completing the characteristics of all themes and sub-themes, data saturation was achieved, and sampling was stopped. This study was approved by the Ethics Committee of Iran University of Medical Sciences (IR.IUMS.REC 1395.9311286011). All participants gave informed written consent. All data were managed and analyzed anonymously. Member check and peer check was conducted to increase credibility.

\section{Results}

Sixteen interviews with 14 psychiatrists were conducted. Interviews lasted between 30 to 55 minutes with an average of 45 minutes. Ten participants were faculty members. Two participants were psychiatrists who worked at university-affiliated hospitals, but they were not faculty members. Two psychiatrists worked exclusively in the private sector. Four participants were female. The Average of participants age was 53 years, and they had an average of 14 years of practice as a psychiatrist.

Our results showed that psychiatrists decide to disclose the diagnosis based on several factors. We summarized these factors in a central theme, passive situational decision making based on paternalism and displacement of responsibility. It has two subthemes, including "passive and situational decision making" and "paternalism and displacement of responsibility." Each theme presented by detailed quotations. The related codes, sub-themes, and themes are presented in Table 1.

Psychiatrists had a passive approach in the disclosure of the diagnosis to their patients. They also considered various factors, such as the patient's condition, for deciding whether to tell a diagnosis or not. This theme has two subthemes, which are passive approach and situational decision making.

Passive approach: Even though telling the diagnosis is an essential part of the treatment process, which should be conducted actively by psychiatrists, they chose a more passive approach. They usually refused to tell diagnoses to patients. However, when the patient questioned them, they revealed the name of the disease to him/her. They argued that knowing the diagnosis does not help patients because it is only a name. They believed that patients should receive complete information. In the current state of the health system, with patients crowding and a shortage of specialized staff, the possibility to provide complete information is very unrealistic. Therefore, they had decided not to take an active approach in the disclosure of the diagnosis to patients.

"I do not usually tell my patients the name of their diseases, the diagnosis is just a name and does not provide much information to the patient. Patients need more information. The name of the disease alone can be misleading."

"If the patient asks for the name of the disease, I will try to provide the needed information."

Situational decision making: Psychiatrists considered factors such as their certainty about the diagnosis, patient insight, how much help is knowing the diagnosis for a patient, and patient condition at the time of diagnosis disclosure. They believed that there was no disruption in the patient's treatment process. They also did not like the patient to become too sensitive to his illness and diagnosis. They also tried to minimize trauma as much as possible.

"It is not helpful to know the diagnosis for a patient who is in an emergency condition or a patient who does not have insight."

"It is possible to disclose a diagnosis to the patient when the therapeutic relationship is formed and telling the diagnosis does not cause an impairment in the treatment process."

Paternalism and displacement of responsibility: Our results showed that psychiatrists had a sense of paternalism towards their patients. The sense of paternalism was accompanied by the avoidance of disclosing the diagnosis. This theme had two sub-themes including a sense of paternalism and displacement of responsibility.

Sense of paternalism: Psychiatrists considered themselves as the guardians of the patients, and they made decisions for their patients. They decided which patient

Table 1. Themes, subtheme, and codes of the psychiatrists' approaches regarding disclosure of psychiatric disorders

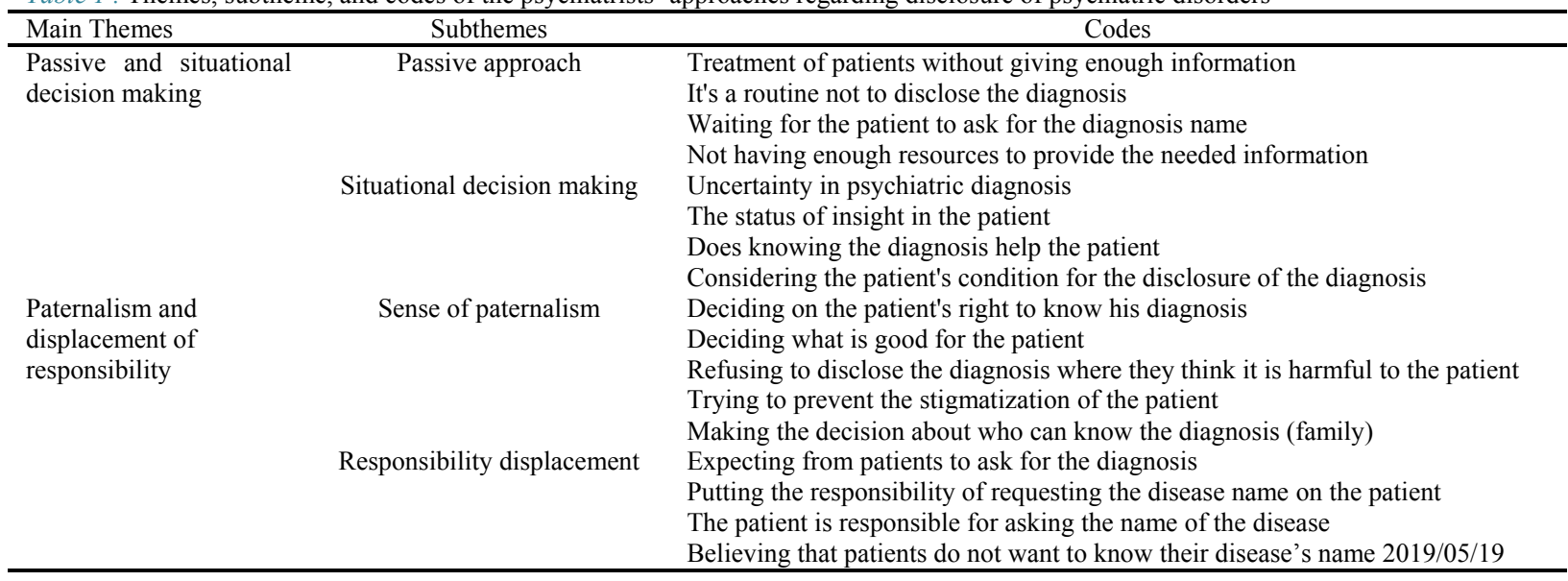


could or should know the diagnosis. They also decided who, including family members, should know about the name of the disease. Psychiatrists decided not to disclose the diagnosis when they thought knowing the diagnosis was harmful to the patient. One of the reasons for not disclosing the diagnosis was to prevent the patient from becoming stigmatized.

"First, I need to see if it helps the patient to know the diagnosis. If the disclosure of the diagnosis is good for him, I will disclose the name of the disease."

"Patients mental health disorders suffer from high levels of stigma. Knowing the name of the disease may cause stigma. If disclosure of diagnosis causes stigma, it should be avoided."

"If I come to the conclusion that disclosing the diagnosis to the family helps the patient, I will give them the information."

Responsibility displacement: Psychiatrists considered patients responsible for asking the name of the disease. If patients did not ask about the name of the disease, they would not receive information regarding the disease name. Some psychiatrists believe that patients with mental health disorders do not like the disease's name to be disclosed to them.

"Usually, I do not disclose the disease until the patient asks about it. If necessary, I will disclose the name of the illness to the family members ... When family members ask, and I know they will help the patient, I'll disclose the diagnosis to them."

"Patients with mental health disorders do not like to know their disease's name. Usually knowing the diagnosis will hurt them."

"Whenever the patient asks me, I will disclose the name of the illness. Perhaps the patient's choices are limited. At the same time, knowing the diagnosis name is not always in the best interest of the patient."

\section{Discussion}

The purpose of this study was to investigate the psychiatrist's experiences of disclosing psychiatric diagnosis. The results of the study showed that psychiatrists consider several factors in their decision of diagnosis disclosure. Passive and situational decision making was the first theme in our findings. Psychiatrists had a passive approach regarding the diagnosis disclosure, which is in contrast with new paradigms in medicine. The new medicine is trying to get patients more involved in treatment. Patients are the center of all activities, especially in decision making. Making an important decision, like who can know about the name of the disease, by physicians' contrasts with putting the patient in the center of the treatment process. In addition, in all clinical guidelines, the disclosure of diagnosis to the patient is considered as the first step in the treatment. However, it does not always seem possible. Based on our findings, one of the main concerns of psychiatrists was the change in their understanding of the nature of the patient's disease over time. The exact diagnosis of psychiatric illness is a time-consuming process. The diagnosis of many patients may change over time which has been shown in numerous studies $(14,15$,
20).

Diagnosis disclosure is a concept of interest in the context of medical ethics in Iran. Also, many studies have been conducted to evaluate the effect of diagnosis disclosure on quality of life and their mental health of patients. Most of these studies were focused on cancer patients. While the results of studies in cancer patients are controversial, we did not find any study in the field of mental illnesses in Iran. In Iran, psychiatric patients are generally considered as incompetent. There are certain rules which deny them from having some authority to protect them from others. However, there is no rule against knowing their diagnosis. The result of the current study also showed that there is no unity in the practice of diagnosis disclosure among psychiatrists.

Situational decision making is one of the most commonly used methods in medicine, which has long been used in clinical settings (11). In fact, it is a requirement of personalized medicine. In this approach, which is a branch of medicine, patient-related factors, such as environmental conditions, are used to prevent, diagnose, and treat diseases (21). This approach has also been used in psychiatry. In this approach, each patient undergoes treatment appropriate to his/her condition. Decision making based on the patient's condition is a type of personalized medicine (1). It can be argued that during the treatment process, psychiatrists make different decisions according to the patient's condition; the diagnosis disclosure is one of them. From this perspective, a psychiatrist's approach may not be passive. But disclosure of diagnosis at the patient's request is more representative of the passive approach.

Several studies have been conducted on the disclosure of diagnosis in various diseases, including psychiatric disorders. The results of a research conducted by Moran, $\mathrm{Oz}$ (16) showed that psychiatrists considered the diagnosis disclosure as to be challenging, unconventional and harmful. They categorized three effective areas in the disclosure of diagnosis, including the nature of the disease, the relationship between the patient / family and the psychiatrist, and the personal problems of the psychiatrist.

Our results showed a sense of paternalism in psychiatrists, which was a barrier to disclosure of diagnosis. The results of previous studies also showed that psychiatrists have stronger feel of paternalism over their patients than other doctors. Being incapable and the possibility of abusing psychiatric patients make psychiatrists more cautious in their management. This paternalistic sense can the principle of autonomy to be compromised at the time of hospitalization $(13,22)$.

The disclosure of diagnosis has its own supporters and opponents. Opponents of the disclosure have diagnostic, moral, and practical reasons for it. The first reason is related to the uncertainty in the diagnosis of psychiatric illnesses. Opponents argue that it's difficult to be completely sure of the name of psychiatric illnesses and require to role out the differential diagnosis (23-25). Morally, opponents argue that knowing the diagnosis can traumatize the patient. Patients may experience bad feelings like fear, reduced self-esteem, and may have adverse reactions like suicide (11). Psychiatrists also consider disclosure of psy- 
chiatric diagnosis as a kind of bad news giving, and they try to protect the patient from it (26). The last reason was the psychiatrist's intention to protect the patient from being exposed to problems such as stigma (24).

Supporters of diagnosis disclosure have reasons like the patient's expectations for receiving information and the truth about health care and the benefits of having such information. From their perspective, knowing the disease's name allows the patient to receive help and seek support sources, reduces patient concerns and they find that there are other people who have the same illness $(27,28)$. Providing information to family members and not disclosing the diagnostic name for the patient is contrary to the patient-centered approach and can lead to negative experiences in the patient. Diagnosis disclosure can also improve the relationship between the physician and the patient, which leads to better management of symptoms and patient treatment. Knowing the name of the disease makes the patient adapt to emotions related to it, such as shame and fear $(27,28)$.

The results of the current study showed that the sense of paternalism in psychiatrists caused that they decide to disclose the diagnosis to the family instead of the patient. This approach shows that psychiatrists consider patients as incapable persons who are unable to manage their diseaserelated knowledge. This result may also indicate that psychiatrists consider the patient's family as a source of support that can help the patient. The result that has been emphasized in earlier studies $(29,30)$.

The most important limitation of the present study was its dependence on the psychiatrist's statements. While the interview is a reliable method in qualitative research, but it cannot show a complete picture of the participants' practice. The actual performance of psychiatrists may be slightly different from what they say. However, in the present study, the primary aim was not an assessment of the performance of psychiatrists. Another important limitation of the current study was that all of the participants had been selected from Iranian culture and the results may be different in other countries, but it is the nature of qualitative research that is context-dependent. We suggest more studies to be done in this area in different cultures. We also propose that the legal aspects of diagnosis disclosure in patients with mental health disorders should be considered further.

\section{Conclusion}

The results of this study showed that psychiatrists did not actively disclose the diagnosis name to patients. Diagnosis disclosure was influenced by several factors, such as the certainty about the diagnosis and the severity of the disease. Avoidance to the disclosure of the diagnosis has become the conventional approach. However, in response to the patients' request, psychiatrists usually revealed the name of the disease.

Diagnosis disclosure based on the patient's request is a medical approach that has been abandoned in the past decades. This passive approach does not respect the patient's rights. The paternalistic nature of this approach causes that psychiatrists consider themselves as responsi- ble for patients' welfare. The prevailing approach in the treatment of psychiatric patients seems to be the same as the medical approach. In this approach, the goal is to reduce the symptoms of the disease and disclosing the diagnosis in this approach is usually by patients' requests. Psychiatry requires different approaches in diagnosis disclosure that have become more common in recent years to reassure the rehabilitation and recovery of patients.

The most important application of the present research is in the treatment and rehabilitation of psychiatric patients. Recovery and rehabilitation require approaches that the patient actively participates in them. Psychiatrists need to acquire and use more skills for giving bad news and reducing stigma. Recovery and rehabilitation approaches can be more successful in this area.

\section{Acknowledgments}

The authors thank all participants who take part in this study and participate in interviews.

\section{Conflict of Interests}

The authors declare that they have no competing interests.

\section{References}

1. Moumjid N, Gafni A, Bremond A, Carrere MO. Shared decision making in the medical encounter: are we all talking about the same thing? Med Decis Making. 2007;27(5):539-46.

2. Sheykh-Talimi M, Shariati-Nasab S, Zare MK, Omani-Samani R. The right to information and their exceptions in medical practices in the Iranian legal system. J Med Ethics Hist Med. 2016;9:15.

3. Abazari P, Taleghani F, Hematti S, Malekian A, Mokarian F, Hakimian SMR, et al. Breaking bad news protocol for cancer disclosure: an Iranian version. J Med Ethics Hist Med. 2017;10:13.

4. Milton AC, Mullan B, Hunt C. Information giving challenges and support strategies at the time of a mental health diagnosis: qualitative views from Australian health professionals. Soc Psychiatry Psychiatr Epidemiol. 2016;51(5):735-46.

5. Zahedi F. The challenge of truth telling across cultures: a case study. J Med Ethics Hist Med. 2011;4:11.

6. Shergill SS, Barker D, Greenberg M. Communication of psychiatric diagnosis. Soc Psychiatry Psychiatr Epidemiol. 1998;33(1):32-8.

7. McDonald-Scott P, Machizawa S, Satoh H. Diagnostic disclosure: a tale in two cultures. Psychol Med. 1992;22(1):147-57.

8. Laugharne R, Priebe S. Trust, choice and power in mental health: a literature review. Soc Psychiatry Psychiatr Epidemiol. 2006;41(11):843-52

9. Laugharne R, Priebe S, McCabe R, Garland N, Clifford D. Trust, choice and power in mental health care: experiences of patients with psychosis. Int J Soc Psychiatry. 2012;58(5):496-504.

10. Wild R, Pettit T. Conspiracy of silence? Telling patients with schizophrenia their diagnosis. Psychiatric Bullet. 2002;26(1):37-

11. Cleary M, Hunt GE, Horsfall J. Delivering difficult news in psychiatric settings. Harv Rev Psychiatry. 2009;17(5):315-21

12. Cleary M, Hunt GE, Walter G. Delivering difficult news. Views of mental health staff in inpatient settings. J Psychosoc Nurs Ment Health Serv. 2010;48(6):32-9.

13. Jantos M. Patient autonomy on a psychiatric ward. Arch Psychiatry Psychother. 2016;3:13-7.

14. Adeponle AB, Groleau D, Kirmayer LJ. Clinician reasoning in the use of cultural formulation to resolve uncertainty in the diagnosis of psychosis. Cult Med Psychiatry. 2015;39(1):16-42.

15. Queirazza F, Semple DM, Lawrie SM. Transition to schizophrenia in acute and transient psychotic disorders. Br J Psychiatry. 2014;204(4):299-305 
16. Moran GS, Oz G, Karnieli-Miller O. Psychiatrists' challenges in considering disclosure of schizophrenia diagnosis in Israel. Qual Health Res. 2014;24(10):1368-80.

17. Moran G, Mashiach-Eizenberg M, Roe D, Berman Y, Shalev A, Kaplan Z, et al. Investigating the anatomy of the helping relationship in the context of psychiatric rehabilitation: The relation between working alliance, providers' recovery competencies and personal recovery. Psychiatry Res. 2014;220(1):592-7.

18. Marzanski M. On telling the truth to patients with dementia. West J Med. 2000;173(5):318-23.

19. Braun V, Clarke V. Using thematic analysis in psychology. Qual Res Psychol. 2006;3(2):77-101.

20. Blázquez A, Ortiz AE, Castro-Fornieles J, Morer A, Baeza I, Martínez E, et al. Five-year diagnostic stability among adolescents in an inpatient psychiatric unit. Compr Psychiatry. 2018.

21. Clayman ML, Gulbrandsen P, Morris MA. A patient in the clinic; a person in the world. Why shared decision making needs to center on the person rather than the medical encounter. Patient Educ Couns. 2017;100(3):600-4.

22. Bentley KJ, Cummings CR, Casey RC, Kogut CP. Professional identity and shared decision making among psychiatry residents: designing a brief teaching module. J Ment Health Train Educ Pract. 2018;13(2):112-23.

23. Arango C, Amador X. Lessons learned about poor insight. Schizophr Bull. 2011;37(1):27-8.

24. Rose D, Thornicroft G. Service user perspectives on the impact of a mental illness diagnosis. Epidemiol Psichiatr Soc. 2010;19(2):140-7.

25. Gallagher A, Arber A, Chaplin R, Quirk A. Service users' experience of receiving bad news about their mental health. J Ment Health. 2010;19(1):34-42.

26. Mitchell AJ. Reluctance to disclose difficult diagnoses: a narrative review comparing communication by psychiatrists and oncologists. Support Care Cancer. 2007;15(7):819-28.

27. Yap M, Reavley N, Jorm A. The associations between psychiatric label use and young people's help-seeking preferences: results from an Australian national survey. Epidemiol Psychiatr Sci. 2014;23(1):51-9.

28. Reavley NJ, Jorm AF. Young people's recognition of mental disorders and beliefs about treatment and outcome: findings from an Australian national survey. Aust N Z J Psychiatry. 2011;45(10):890-8.

29. Caqueo-Urizar A, Rus-Calafell M, Urzua A, Escudero J, GutierrezMaldonado J. The role of family therapy in the management of schizophrenia: challenges and solutions. Neuropsychiatr Dis Treat. 2015;11:145-51.

30. Hsiao CY, Tsai YF. Factors of caregiver burden and family functioning among Taiwanese family caregivers living with schizophrenia. J Clin Nurs. 2015;24(11-12):1546-56. 